\title{
ANALYSIS OF PEAK TORQUE OF WOMEN TREATMENT CHEMOTHERAPY WITH APPARENTLY HEALTHY WOMEN
}

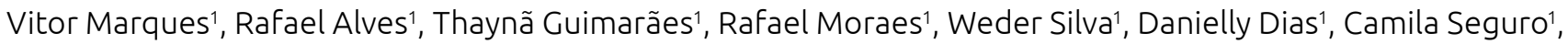
Carlos Vieira'

${ }^{1}$ Faculty of Physical Education and Dance, Universidade de Goiás - Goiânia (GO), Brazil.

Objective: To evaluate and compare the muscular performance of women with breast cancer during chemotherapy with healthy women. Methods: Participated the study 37 women divided into a control group (age: 52,2 $\pm 13,11$ ) composed of healthy women and group treatment (age: 55,8 $\pm 8,37$ ) composed of women who were between the third and fourth cycle of chemotherapy. The muscular performance was evaluated through the isokinetic dynamometer biodex system III, to which the peak torque was analyzed by performing 2 sets of 4 repetitions of knee extension at $60^{\circ} \cdot \mathrm{s}^{-1}$ in the concentric action, with 2-minute interval between the set. The normality of the data was evaluated by the Shapiro-Wilk test. Data were analyzed by Student's $t$ test. The significance level was defined a priori at $\mathrm{p}<0.05$. The size of the effect $d$ of Cohen was calculated from the difference between the groups to examine the magnitude of the effect of breast cancer treatment on the variables investigated. The present study was approved by the Research Ethics Committee of the Federal University of Goiás (CAAE: 50717115.4.0000.5083), and by the Research Ethics Committee of the Hospital of Clinics (HC / UFG) (CAAE: 50717115.4.3001.5078). Resolution 466/12 of the National Health Council. Results: There was no significant difference in peak torque between groups ( $\mathrm{p}=0.95, \mathrm{~d}=0.02$ ). Conclusion: Women in breast cancer treatment during the third and fourth cycles of chemotherapy did not present differences in measures of muscular performance when compared to healthy women. 